\title{
Tale of two vaccines: differences in response to herpes zoster vaccines
}

\author{
Anne A. Gershon \\ Columbia University College of Physicians and Surgeons, New York, New York, USA.
}

\begin{abstract}
About one-third of the US population will develop herpes zoster $(\mathrm{HZ}$, commonly known as shingles) over a lifetime, while two-thirds will not. It is not clear exactly why certain people are susceptible to $\mathrm{HZ}$; however, we may be coming closer to an answer. In this issue of the $J C I$, a study by Levin et al. provides important details concerning pathogenesis of and protection from $\mathrm{HZ}$. The authors characterized differences in the immunologic responses induced by two $\mathrm{HZ}$ vaccines, the live attenuated zoster vaccine (ZV) and the more recently developed adjuvanted varicellazoster virus (VZV) glycoprotein E (gE) subunit herpes zoster vaccine ( $\mathrm{HZ}$ / su), in vaccine-naive subjects and those previously vaccinated with $\mathrm{HZ}$. The observed differences in responses paralleled the observed clinical protection of the two zoster vaccines, with $\mathrm{HZ} /$ su being superior to $\mathrm{HZ}$. Together, these results seem to explain immunologically why the new subunit vaccine outperforms the live vaccine. These differences may also provide clues as to why $\mathrm{HZ}$ develops in the first place.
\end{abstract}

\section{Varicella-zoster virus: two diseases, one pathogen}

Varicella-zoster virus (VZV) is a herpesvirus that causes two diseases. The primary infection varicella (also known as chickenpox) is characterized by a blister-like rash all over the body, malaise, and fever. Initial disease results in lifelong latent infection of peripheral neurons with VZV. Latent VZV can reactivate, often in the setting of old age or immunosuppression, resulting in a second, usually localized unilateral infection referred to as herpes zoster (HZ, commonly known as shingles). About onethird of the US population will develop $\mathrm{HZ}$ during a lifetime; however, it is not fully understood why certain people are susceptible to HZ and others are not. Vaccines against VZV first became available about 35 years ago, following the development of a live attenuated virus vaccine by Takahashi et al. (1) that was designed to prevent varicella. Many investigators were initially critical of this live vaccine because VZV is a herpesvirus and viral latency is known universally to follow herpesvirus infections. Moreover, no vaccine that causes latent infection had ever been administered to humans. The live attenuated varicella vaccine was shown to be safe and even lifesaving in children with underlying leukemia; therefore, resistance to the varicella vaccine diminished (2). Latency did not seem to be a problem and might even contribute to immunity against varicella, as intermittent silent reactivation of $\mathrm{VZV}$ appears to stimulate immunity to varicella $(3,4)$.

The licensure of the live attenuated varicella vaccine led directly to development of the first live attenuated zoster vaccine $(\mathrm{ZV})$ for prevention of $\mathrm{HZ}$, which was licensed for use in the US in 2005 (5). The dose of live virus necessary to stimulate the immune system in persons over 50 years of age was 14 times greater than the dose used for prevention of varicella in children or adults (5). The rationale for the $\mathrm{ZV}$ vaccine was to stimulate cell-mediated immu-

Related Article: p. 4429

Conflict of interest: AAG has a laboratory service contract with Merck regarding safety of live VZV vaccines.

Reference information: / Clin Invest. 2018;128(10):4245-4247. https://doi.org/10.1172/JCI123217.

nity (CMI) in aged populations. ZV was a remarkable proof of concept of a successful vaccine against $\mathrm{HZ}$, as waning immunity to VZV could be boosted successfully in the elderly and thus prevent disease due to reactivation of VZV. Unfortunately, the efficacy of the $\mathrm{ZV}$ vaccine is dependent on the age of the individual at the time of administration, with efficacy demonstrably less for 70-year-old subjects than for 60-year-old subjects. The age-dependent efficacy of ZV was a concern because the primary reason for development of that vaccine was the prevention of postherpetic neuralgia (PHN) (5), which can be devastating, particularly in the elderly, and increases in incidence with advancing age. Another vaccine was then developed to prevent HZ. Unlike ZV, the new VZV glycoprotein $\mathrm{E}$ (gE) subunit herpes zoster vaccine $(\mathrm{HZ} / \mathrm{su})$, which was licensed just this year, is not infectious and is composed of the major antigen of $\mathrm{VZV}, \mathrm{gE}$, and a relatively new adjuvant, ASO1B, that boosts both innate and adaptive immunity $(6,7)$. AS01B acts locally in lymph nodes near the injection site and does not depend on circulation in the body (8).

Remarkably, the HZ/su is able to prevent $\mathrm{HZ}$ and $\mathrm{PHN}$ in 70- to 80-year-old patients. In fact, the adjuvanted $\mathrm{HZ} / \mathrm{su}$ has outperformed the live attenuated $\mathrm{ZV}$ in all age groups in which it was tested (6, 7, 9). As with any new vaccine, an important aspect of $\mathrm{HZ} / \mathrm{su}$ research will be continued monitoring of effectiveness in preventing $\mathrm{HZ}$ with time after vaccination.

\section{Understanding different responses to $\mathrm{ZV}$ and $\mathrm{HZ} / \mathrm{su}$}

In this issue, Levin et al. employed standard tests of CMI (FloroSpot, also known as ELISpot) (10-14) and developed immunologic flow cytometric methods that allowed them to identify significant differences in the immunologic responses that result in response to $\mathrm{ZV}$ and $\mathrm{HZ} / \mathrm{su}$ (15). Moreover, the flow cytometry methods enabled the investigators to identify 


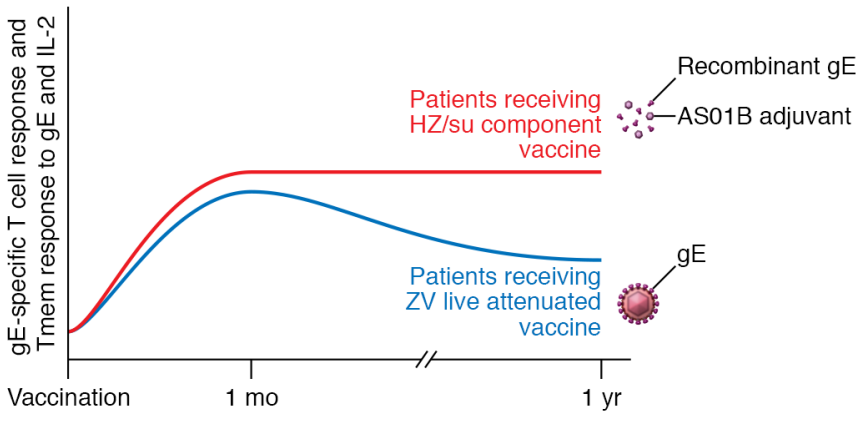

Figure 1. Live attenuated and adjuvanted component vaccines for $\mathrm{HZ}$ elicit different responses. Both vaccine-naive subjects and those that have received the live attenuated $Z V$ vaccine more than 5 years ago exhibit a low baseline response to the VZV gE. Following immunization with ZV, subjects had a measurable increase in $\mathrm{gE}$-specific $\mathrm{T}$ cell response and memory $\mathrm{T}$ cell (Tmem) response to gE and IL-12 at 1 month. In contrast, subjects that received the adjuvanted component vaccine $\mathrm{HZ} / \mathrm{su}$ had a marked increase in gE-specific T cell response and memory T cell response to gE and IL-12 at 1 month after vaccination. One year after vaccination, $T$ cell responses were minimal in subjects that received $\mathrm{ZV}$; however, these responses were sustained in patients that received $\mathrm{HZ} / \mathrm{su}$.

significant differences in the immunologic responses resulting from the two $\mathrm{HZ}$ vaccines. Levin and colleagues evaluated CMI responses in healthy vaccine-naive 50- to 85-year-old individuals following vaccination with ZV or HZ/su. Responses to HZ/su boost were also evaluated in an additional cohort of subjects that had previously been immunized with $\mathrm{ZV}$. T cell responses against VZV gE were usually low, but present, in subjects that had not previously been vaccinated and those that had previously been vaccinated with ZV. Given the age of the vaccine-naive participants, they almost certainly had varicella at some point prior to the study. In all cases, subjects vaccinated with $\mathrm{HZ} /$ su developed $\mathrm{gE}$ specific $\mathrm{T}$ cell responses that were as much as ten times higher than those induced by $\mathrm{ZV}$ and measurable as long as 12 months after immunization. Memory $\mathrm{T}$ cell responses to gE and IL-2 were also higher in response to $\mathrm{HZ} / \mathrm{su}$ than in response to ZV. Peak memory responses (PMR) to IL-2 were observed 1 month after vaccination (after 1 dose for $\mathrm{ZV}$ and 2 doses for $\mathrm{HZ} / \mathrm{su}$ ) and were found necessary for persistence of the Th1 responses. This detailed immunologic comparison between the response to the highly effective HZ/su (roughly $97 \%$ clinical protection) and the less effective $\mathrm{ZV}$ vaccine (roughly $60 \%$ clinical protection) provides a potentially new understanding of specific immunity prior to development of HZ. In recipients of the adjuvanted $\mathrm{HZ} / \mathrm{su}$, memory $\mathrm{T}$ cells persisted more efficiently than in recipients of $\mathrm{ZV}$. The AS01B adjuvant, which is also somewhat novel, is almost certainly critical for the strong immunologic response to $\mathrm{HZ} / \mathrm{su}$. Memory T cells for gE and/or IL-2, which may be low in individuals at high risk of developing $\mathrm{HZ}$, thus may be critical factors that predispose individuals to $\mathrm{HZ}$ development. If further validated, these phenomena could be useful for evaluating vaccine efficacy in individual patients as markers in future vaccine studies and also might provide important information on the pathogenesis of HZ (Figure 1).

Not only is HZ/su more effective in preventing $\mathrm{HZ}$ than $\mathrm{ZV}$, but it is not infectious. The attenuated $\mathrm{ZV}$ is able to multiply significantly in some immunocomand death (16). $\mathrm{ZV}$ is not recommended for immunocompromised patients, but on occasion has been inadvertently administered to them, resulting in rare serious consequences $(16,17)$.

\section{VZV latency and $\mathrm{HZ}$ manifestation}

It is not fully understood how VZV latency occurs and is maintained. Latency occurs in peripheral neurons and may involve those of the dorsal root and cranial nerve ganglia, which would explain the resulting unilateral painful $\mathrm{HZ}$ skin rash, and those of the autonomic nervous system, including the enteric nervous system (ENS), which may not result in production of a rash. Therefore, $\mathrm{HZ}$ in these cases would be difficult to diagnose. VZV reactivation promised hosts and cause serious illness in the ENS may result in a wide variety of gastrointestinal manifestations, including ulcers of the colon, intestine, and stomach, achalasia, and esophagitis (18).

During latency, viral DNA is circularized and neurons appear to be normal. Viral expression proceeds in a cascade of VZV DNA synthesis, and when this cascade is somehow blocked, the virus is said to be latent (19). Exactly how much viral expression occurs during latency is somewhat controversial and is being widely investigated. Reactivation of VZV may be symptomatic or asymptomatic (19-21). The phenomenon of asymptomatic reactivation, which can be indicated by the temporary presence of noninfectious VZV DNA in saliva, suggests that VZV reactivation that leads to disease manifestation may be a two-step process (1, 21). First, when reactivation does occur, cellular immunity quickly interferes with full viral synthesis and there are no symptoms. Second, when cellular immunity is unable to suppress reactivated VZV, symptoms appear as the virus multiplies. Eventually, in most people, cellular immune responses are able to regain control of VZV multiplication and symptoms resolve. The exact cellular immune responses responsible for controlling emergence from latency and recovery are now being clarified. The newly described responses by Levin, as measured by flow cytometry, seem to shed light on these processes and suggest that when the body can rapidly mobilize memory Th1 cells that produce IL-2, reactivating VZV can be suppressed before symptoms begin. As stated in their paper, Levin et al. plan to prospectively investigate this possibility in immunocompromised patients in a future study of HZ/su (15). Presumably, such a study will include vaccinees in whom important immune responses fail to occur or persist and who then develop HZ. If the suspected scenario regarding cellular immune responses occurs, it will not only yield practical information about success of vaccination, but also represent a significant advance in our understanding of $\mathrm{HZ}$ pathogenesis.

\section{Clinical implications and future directions}

When investigating the efficacy of a vaccine, it is extremely helpful to have a laboratory study-validated immune correlate to indicate whether a vaccine will be effec- 
tive in preventing disease. For example, when the varicella vaccine was being evaluated, an antibody test termed fluorescent antibody to membrane antigen (FAMA) was available. The presence of FAMA in blood reliably predicted that vaccinees would be protected from varicella if they were exposed to the infection $(22,23)$. Unlike the case for $\mathrm{HZ}$ vaccines, it was not necessary to follow large cohorts of individuals for years to evaluate varicella vaccine efficacy (although this was done). Not many reliable immune correlates exist for vaccines; therefore, an immune correlate that indicates $\mathrm{HZ}$ vaccine efficacy would obviously be useful for evaluating both initial responses and the length of time after vaccination for which they are efficacious. At present, $\mathrm{HZ}$ can occur in patients that have received $\mathrm{ZV}$ and/or $\mathrm{HZ} / \mathrm{su}$, and currently, the only way to judge efficacy is to follow vaccinees for many years to determine whether or not they develop HZ. Such long-term evaluation is also used to determine for how long the immunity lasts and whether booster vaccine doses are necessary. In previous studies, no particular level of CMI, as measured by ELISpot, was shown to be indicative of protection (24). It will be an exciting development if some of the assays described by Levin et al. are able to be utilized as true immune correlates, such as memory Th1/IL-2 responses and possibly gE antibody levels. Such validation should be possible because protective immunity, as described by Levin et al., can be followed prospectively for a relatively short term in immunocompromised patients following immunization with $\mathrm{HZ} /$ $\mathrm{su}$, which is not infectious. We look forward to the next installment of this exciting story of vaccination to prevent HZ.

\section{Acknowledgments}

AGG is supported by NIH R01DK093094.

Address correspondence to: Anne A. Gershon, Columbia University Vagelos College of Physicians and Surgeons, 630 West 168th Street, New York, New York 10032, USA. Phone: 212.305.9445; Email: aag1@ cumc.columbia.edu.

1. Takahashi M, Otsuka T, Okuno Y, Asano Y, Yazaki T. Live vaccine used to prevent the spread of varicella in children in hospital. Lancet. 1974;2(7892):1288-1290.

2. Gershon AA, et al. Live attenuated varicella vaccine. Efficacy for children with leukemia in remission. JAMA. 1984;252(3):355-362.

3. Hope-Simpson RE. The nature of herpes zoster: a long-term study and a new hypothesis. Proc $R$ Soc Med. 1965;58:9-20.

4. Lopez AS, Zhang J, Marin M. Epidemiology of varicella during the 2 -dose varicella vaccination program - United States, 2005-2014. MMWR Morb Mortal Wkly Rep. 2016;65(34):902-905.

5. Oxman MN, et al. A vaccine to prevent herpes zoster and postherpetic neuralgia in older adults. NEngl J Med. 2005;352(22):2271-2284.

6. Lal H, et al. Efficacy of an adjuvanted herpes zoster subunit vaccine in older adults. $N$ Engl J Med. 2015;372(22):2087-2096.

7. Cunningham AL, et al. Efficacy of the herpes zoster subunit vaccine in adults 70 years of age or older. N Engl J Med. 2016;375(11):1019-1032.

8. Didierlaurent AM, Laupèze B, Di Pasquale A, Hergli N, Collignon C, Garçon N. Adjuvant system AS01: helping to overcome the challenges of modern vaccines. Expert Rev Vaccines. 2017;16(1):55-63

9. Cunningham AL. The herpes zoster subunit vaccine. Expert Opin Biol Ther. 2016;16(2):265-271.

10. Smith JG, et al. Measurement of cell-mediated immunity with a varicella-zoster virus-specific interferon- $\gamma$ ELISPOT assay: responses in an elderly population receiving a booster immunization. JMed Virol. 2003;70(suppl 1):S38-S41.

11. Levin MJ, et al. Cellular and humoral responses to a second dose of herpes zoster vaccine administered 10 years after the first dose among older adults. J Infect Dis. 2016;213(1):14-22.
12. Weinberg A, et al. Varicella-zoster virus-specific cellular immune responses to the live attenuated zoster vaccine in young and older adults. Jimmunol. 2017;199(2):604-612.

13. Weinberg A, Levin MJ. VZV T cell-mediated immunity. In: Abendroth A, Arvin AM, Moffat JF, eds. Varicella-Zoster Virus. Vol. Current Topics In Microbiology And Immunolog. Vol. 342. Berlin, Germany: Springer, 2010:341-357.

14. Weinberg A, et al. Varicella-zoster virus-specific immune responses to herpes zoster in elderly participants in a trial of a clinically effective zoster vaccine. J Infect Dis. 2009;200(7):1068-1077.

15. Levin MJ, et al. Th1 memory differentiates recombinant from live herpes zoster vaccines. J Clin Invest. 2018;128(10):4429-4440.

16. Bhalla P, et al. Disseminated, persistent, and fatal infection due to the vaccine strain of varicella-zoster virus in an adult following stem cell transplantation. Clin Infect Dis. 2015;60(7):1068-1074.

17. Willis ED, et al. Herpes zoster vaccine live: A 10 year review of post-marketing safety experience. Vaccine. 2017;35(52):7231-7239.

18. Gershon AA, Chen J, Gershon MD. Use of saliva to identify varicella zoster virus infection of the gut. Clin Infect Dis. 2015;61(4):536-544

19. Arvin A, Gilden D. Varicellar-zoster virus. In: Knipe D, Howley P, eds. Fields Virology. Philadelphia, Pennsylvania, USA: Lippincott, Williams, and Wilkins; 2013:2015-2057.

20. Cohrs RJ, Mehta SK, Schmid DS, Gilden DH, Pierson DL. Asymptomatic reactivation and shed of infectious varicella zoster virus in astronauts. JMed Virol. 2008;80(6):1116-1122.

21. Mehta SK, Cohrs RJ, Forghani B, Zerbe G, Gilden DH, Pierson DL. Stress-induced subclinical reactivation of varicella zoster virus in astronauts. J Med Virol. 2004;72(1):174-179.

22. Gershon AA, Steinberg S, Brunell PA. Zoster immune globulin. A further assessment. $N$ Engl J Med.1974;290(5):243-245.

23. Williams V, Gershon A, Brunell PA. Serologic response to varicella-zoster membrane antigens measured by direct immunofluorescence. J Infect Dis. 1974;130(6):669-672.

24. Levin MJ, et al. Varicella-zoster virus-specific immune responses in elderly recipients of a herpes zoster vaccine. J Infect Dis. 2008;197(6):825-835 